\title{
Long-term dietary nitrite and nitrate deficiency causes the metabolic syndrome, endothelial dysfunction and cardiovascular death in mice
}

\author{
Mika Kina-Tanada ${ }^{1,2} \cdot$ Mayuko Sakanashi $^{1} \cdot$ Akihide Tanimoto $^{3} \cdot$ Tadashi Kaname $^{4}$. \\ Toshihiro Matsuzaki $^{1}$ - Katsuhiko Noguchi ${ }^{1}$ - Taro Uchida ${ }^{1}$ • Junko Nakasone ${ }^{1}$. \\ Chisayo Kozuka $^{5}$ - Masayoshi Ishida ${ }^{1,6}$ - Haruaki Kubota ${ }^{1} \cdot$ Yuji Taira $^{1}$ • \\ Yuichi Totsuka $^{1}$ • Shin-ichiro Kina ${ }^{2}$ - Hajime Sunakawa ${ }^{2} \cdot J_{u n i c h i}$ Omura ${ }^{7}$. \\ Kimio Satoh $^{7}$ - Hiroaki Shimokawa ${ }^{7}$ - Nobuyuki Yanagihara ${ }^{8} \cdot$ Shiro Maeda $^{4}$. \\ Yusuke Ohya $^{9}$ - Masayuki Matsushita ${ }^{10}$ - Hiroaki Masuzaki ${ }^{5}$ Akira Arasaki ${ }^{2}$. \\ Masato Tsutsui ${ }^{1}$
}

Received: 16 September 2016 / Accepted: 27 February 2017 /Published online: 28 March 2017

(C) Springer-Verlag Berlin Heidelberg 2017

\begin{abstract}
Aims/hypothesis Nitric oxide (NO) is synthesised not only from L-arginine by NO synthases (NOSs), but also from its inert metabolites, nitrite and nitrate. Green leafy vegetables are abundant in nitrate, but whether or not a deficiency in dietary nitrite/nitrate spontaneously causes disease remains to be clarified. In this study, we tested our hypothesis that long-term dietary nitrite/nitrate deficiency would induce the metabolic syndrome in mice.

Methods To this end, we prepared a low-nitrite/nitrate diet (LND) consisting of an amino acid-based low-nitrite/nitrate chow, in which the contents of L-arginine, fat, carbohydrates,
\end{abstract}

Mika Kina-Tanada and Mayuko Sakanashi contributed equally to this work.

Electronic supplementary material The online version of this article (doi:10.1007/s00125-017-4259-6) contains peer-reviewed but unedited supplementary material, which is available to authorised users.

Masato Tsutsui

tsutsui@med.u-ryukyu.ac.jp

1 Department of Pharmacology, Graduate School of Medicine, University of the Ryukyus, 207 Uehara, Nishihara, Okinawa 903-0215, Japan

2 Department of Oral and Maxillofacial Functional Rehabilitation, University of the Ryukyus, Okinawa, Japan

3 Department of Pathology, Kagoshima University Graduate School of Medical and Dental Sciences, Kagoshima, Japan

4 Department of Advanced Genomic and Laboratory Medicine, Graduate School of Medicine, University of the Ryukyus, Okinawa, Japan protein and energy were identical with a regular chow, and potable ultrapure water. Nitrite and nitrate were undetectable in both the chow and the water.

Results Three months of the LND did not affect food or water intake in wild-type C57BL/6J mice compared with a regular diet (RD). However, in comparison with the RD, 3 months of the LND significantly elicited visceral adiposity, dyslipidaemia and glucose intolerance. Eighteen months of the LND significantly provoked increased body weight, hypertension, insulin resistance and impaired endothelium-dependent relaxations to acetylcholine, while 22 months of the LND significantly led to death mainly due to cardiovascular disease, including acute
Second Department of Internal Medicine, Graduate School of Medicine, University of the Ryukyus, Okinawa, Japan

6 Regenerative Medicine Research Center, Graduate School of Medicine, University of the Ryukyus, Okinawa, Japan

7 Department of Cardiovascular Medicine, Tohoku University Graduate School of Medicine, Sendai, Japan

8 Department of Pharmacology, School of Medicine, University of Occupational and Environmental Health, Kitakyushu, Japan

9 Third Department of Internal Medicine, Graduate School of Medicine, University of the Ryukyus, Okinawa, Japan

10 Department of Physiology, Graduate School of Medicine, University of the Ryukyus, Okinawa, Japan 
myocardial infarction. These abnormalities were reversed by simultaneous treatment with sodium nitrate, and were significantly associated with endothelial NOS downregulation, adiponectin insufficiency and dysbiosis of the gut microbiota. Conclusions/interpretation These results provide the first evidence that long-term dietary nitrite/nitrate deficiency gives rise to the metabolic syndrome, endothelial dysfunction and cardiovascular death in mice, indicating a novel pathogenetic role of the exogenous NO production system in the metabolic syndrome and its vascular complications.

Keywords Acute myocardial infarction - Cardiovascular death $\cdot$ Diet $\cdot$ Endothelial dysfunction $\cdot$ Metabolic syndrome Mice $\cdot$ Nitrate $\cdot$ Nitric oxide $\cdot$ Nitrite

$\begin{array}{ll}\text { Abbreviations } \\ \text { AMPK } & \begin{array}{l}\text { Adenosine monophosphate-activated protein } \\ \text { kinase }\end{array} \\ \text { CT } & \begin{array}{l}\text { Computed tomography } \\ \text { eNOS }\end{array} \\ \text { Endothelial nitric oxide synthase } \\ \text { EWAT } & \begin{array}{l}\text { Epididymal white adipose tissue } \\ \text { GAPDH }\end{array} \\ \text { Glyceraldehyde-3-phosphate dehydrogenase } \\ \text { iNOS } & \text { Inducible nitric oxide synthase } \\ \text { LND } & \text { Low-nitrite/nitrate diet } \\ \text { nNOS } & \text { Neuronal nitric oxide synthase } \\ \text { NOS } & \text { Nitric oxide synthase } \\ \text { PPAR- } \gamma & \text { Peroxisome proliferator-activated receptor- } \gamma \\ \text { RD } & \text { Regular diet } \\ \text { WAT } & \text { White adipose tissue } \\ \text { WT } & \text { Wild-type }\end{array}$

\section{Introduction}

The metabolic syndrome is defined as a constellation of interrelated cardiovascular risk factors of metabolic origin, including visceral obesity, dyslipidaemia, hypertension, glucose intolerance and insulin resistance [1]. The metabolic syndrome is highly prevalent in industrial countries worldwide, and it has been reported that, in accordance with the most recent harmonised definition of the metabolic syndrome, $23 \%$ of the adult population ( $\geq 20$ years of age) in the USA suffered from the metabolic syndrome in 2009-2010 [2, 3]. Greater global industrialisation is associated with rising rates of obesity, which are expected to dramatically increase the prevalence of the metabolic syndrome worldwide, especially as the population ages [1]. The metabolic syndrome is associated with increased risks of myocardial infarction, stroke, cardiovascular disease mortality and all-cause mortality [4]. It also confers higher risks of peripheral vascular disease, type 2 diabetes, renal disease, hepatic disease and cancer [5-9]. Several factors, including excessive food energy intake, lack of physical activity, genetic susceptibility and ageing, have been thought to be involved in the pathogenesis of the metabolic syndrome. However, the precise mechanisms in its development remain to be fully elucidated [1].

Nitric oxide (NO) exerts multiple biological actions, and is one of the most crucial signalling molecules in mammalian physiology and pathology [10-15]. It is endogenously synthesised from a precursor L-arginine by a family of NO synthases (neuronal [nNOS], inducible [iNOS] and endothelial [eNOS]) with stoichiometric production of L-citrulline. NO has a very short half-life of several seconds and is rapidly oxidised to nitrite $\left(\mathrm{NO}_{2}{ }^{-}\right)$and subsequently to nitrate $\left(\mathrm{NO}_{3}{ }^{-}\right)$. Although nitrite and nitrate were in the past regarded as mere inert metabolites of $\mathrm{NO}$, recent studies have revealed that nitrate is reduced to nitrite and then to NO, so they serve as NO donors [16-18]. Green leafy vegetables, such as spinach and lettuce, and beetroot, are abundant in nitrate, and vegetables are the dominant source of dietary nitrate in humans, contributing to $60-80 \%$ of dietary nitrate intake $[19,20]$. Potable tap water also contains nitrate and a small quantity of nitrite, and $15-20 \%$ of dietary nitrate intake is derived from tap water [19, 20]. It has been reported that cardiac and hepatic ischaemia-reperfusion injury in mice [21-23], cardiac allograft rejection in rats [23] and platelet aggregation in mice [24] are exacerbated by a lownitrite/nitrate diet (LND) (a commercially available low-nitrite/ nitrate chow plus potable ultrapure water, or the low-nitrite/ nitrate chow alone) compared with a regular diet (RD). These results suggest that dietary nitrite/nitrate deficiency modulates disease conditions. Whether or not this deficiency spontaneously causes disease, however, remains to be clarified. In this study, we tested our hypothesis that long-term dietary nitrite/nitrate deficiency would give rise to the metabolic syndrome in mice.

\section{Methods}

Mice This study was approved by the Animal Care and Use Committee, University of the Ryukyus, Japan, and was carried out according to the Institutional Policy on the Care and Use of the Laboratory Animals. The experiments were performed in 6-week-old male wild-type (WT) C57BL/6J mice (Kyudo, Tosu, Japan). All the mice were maintained in temperature- and humidity-controlled rooms illuminated from 08:00 h to 20:00 h. Food and water intake was measured by placing the animals in metabolic cages for $24 \mathrm{~h}$ (see electronic supplementary material [ESM] Methods for further details).

Diet We prepared a purified amino acid-based low-nitrite/nitrate chow in which the contents of L-arginine, fat, carbohydrates, protein and energy were identical to a regular chow (Purina 5001; LabDiet, St Louis, MO, USA), and potable ultrapure Milli-Q water in which nitrite and nitrate levels were 
undetectable (Merck Millipore, Darmstadt, Germany) (Tables 1 and 2). We randomly assigned the mice to diet groups, and either the low-nitrite/nitrate chow plus ultrapure water (LND) or the regular chow plus tap water (RD) was fed ad libitum to the mice from 6 weeks of age for 1.5-22 months (see ESM Methods).

Nitrite and nitrate levels The nitrite and nitrate contents of the chows were analysed using the diazotisation method and the cadmium reduction-diazotisation method, respectively (Japan Food Research Laboratories, Tokyo, Japan). The nitrite and nitrate levels in the plasma and drinking water were assessed using the HPLC-Griess system (ENO-20; Eicom, Kyoto, Japan) (see ESM Methods).

Blood pressure Systolic blood pressure was measured by the tail-cuff method under conscious conditions in a blinded manner (Model MK-2000; Muromachi Kikai, Tokyo, Japan) (see ESM Methods).

Glucose tolerance test Glucose $1 \mathrm{~g} / \mathrm{kg}$ body weight was intraperitoneally injected into the mice under general anaesthesia with sodium pentobarbital $(50 \mathrm{mg} / \mathrm{kg}$, i.p.; Sigma-Aldrich, St Louis, MO, USA) after $18 \mathrm{~h}$ of fasting. Whole blood samples were collected from the tail, and blood glucose levels were evaluated using a portable blood glucose analyser (Glucocard MyDia; Arkray, Kyoto, Japan) (see ESM Methods).

Insulin tolerance test and plasma insulin levels The mice received $0.3 \mathrm{U} / \mathrm{kg}$ body weight of insulin (soluble human insulin, Humulin R; Eli Lilly, Indianapolis, IN, USA) injected into the intraperitoneal cavity under general anaesthesia with sodium pentobarbital $(50 \mathrm{mg} / \mathrm{kg}$, i.p.). The fasting plasma insulin levels were assessed using a commercially available ELISA kit (AKRIN-031; Shibayagi, Gunma, Japan) (see ESM Methods).

Visceral fat weight After euthanasia, epididymal white adipose tissue (EWAT) was removed and weighed (see ESM Methods).

Table 1 Composition of regular and low-nitrite/nitrate chow

\begin{tabular}{lll}
\hline Constituent & Regular chow & Low-nitrite/nitrate chow \\
\hline Protein $(\% \mathrm{~kJ})$ & 28.5 & 28.5 \\
Fat $(\% \mathrm{~kJ})$ & 13.5 & 13.5 \\
Carbohydrate $(\% \mathrm{~kJ})$ & 58.0 & 58.0 \\
L-arginine $(\mathrm{g} / \mathrm{kg})$ & 14.1 & 14.1 \\
Nitrite $(\mu \mathrm{mol} / \mathrm{kg})$ & $<6.5$ & $<6.5$ \\
Nitrate $(\mu \mathrm{mol} / \mathrm{kg})$ & 548.3 & $<4.8$ \\
\hline
\end{tabular}

Values are expressed as the mean values of two samples
Table 2 Nitrate and nitrite levels in tap water and ultrapure water

\begin{tabular}{lll}
\hline Constituent & Tap water & Ultrapure water \\
\hline Nitrite $(\mu \mathrm{mol} / \mathrm{l})$ & 3.30 & $<0.01$ \\
Nitrate $(\mu \mathrm{mol} / \mathrm{l})$ & 6.07 & $<0.01$ \\
\hline
\end{tabular}

Values are expressed as the mean values of two samples

Adipocyte hypertrophy and inflammation Epididymal and peri-renal white adipose tissue (WAT) was stained with an H\&E solution. The circumferential length of each adipocyte was measured using a light microscope equipped with a CCD camera and morphometric analysis software (DS-RilCCD camera and NIS-Elements D 3.2 software; Nikon, Tokyo, Japan). To evaluate inflammation in the adipose tissues, aggregates of inflammatory cells (inflammatory foci consisting of more than ten inflammatory cells) were counted in the maximal cut surface of the EWAT and peri-renal WAT sections on a light microscope at $\times 40$ magnification (see ESM Methods).

Plasma lipid profile Plasma lipid profile was assessed using a Dri-Chem autoanalyser (FDC4000; Fuji Film, Tokyo, Japan). Plasma LDL-cholesterol levels were determined by HPLC (Skylight Biotech, Akita, Japan) [25] (see ESM Methods).

Western blot analysis Western blot analysis was performed as previously reported [26] to detect nNOS, iNOS and eNOS, phosphorylated eNOS at serine 1177 and at threonine 495 (BD Transduction Laboratories, Franklin Lakes, NJ, USA), adiponectin, peroxisome proliferator-activated receptor- $\gamma$ (PPAR- $\gamma$ ), adenosine monophosphate-activated protein kinase (AMPK), sirtuin 1 (Cell Signaling Technology, Danvers, MA, USA), p-AMPK (Santa Cruz Biotechnology, Dallas, TX, USA) and glyceraldehyde-3-phosphate dehydrogenase (GAPDH) (Sigma-Aldrich) (see ESM Methods). The validation of antibodies was performed by the supplier.

Serum cytokine and chemokine levels Serum cytokine/ chemokine levels were measured using a Bio-Plex system (23-Plex, M60-009RDPD; Bio-Rad, CA, USA) [27].

16S ribosomal RNA gene sequencing The faecal DNA samples were run through a next-generation sequencer, as previously reported [28]. The number of each bacterial strain contained in the faecal contents was estimated as the genome equivalent by quantitative real-time PCR of $16 \mathrm{~S}$ ribosomal RNA genes, followed by pyrosequencing of the 16S amplicons [28] (see ESM Methods).

Organ chamber experiment Thoracic aortic rings were mounted in microtissue organ bath chambers (MTBO-1; Labo Support, Osaka, Japan) filled with Krebs-Henseleit 
solution, and the isometric contractile force of the rings was measured (see ESM Methods).

Micro-computed tomography imaging Mice were anesthetised with $2 \%$ isoflurane (Wako Pure Chemical Industries, Osaka, Japan), and computed tomography (CT) images were acquired by three-dimensional micro-CT (R_mCT2; Rigaku Corporation, Tokyo, Japan) (see ESM Methods).

Statistical analyses Most of our results are expressed as mean \pm SEM. Statistical analyses were performed using a Student's $t$ test, or ANOVA followed by Bonferroni's post hoc test. The results of sequencing reads of gut bacteria are expressed as the median and interquartile range, and the statistical analysis was carried out using a Wilcoxon rank-sum test [29]. Kaplan-Meier survival curves were compared using the logrank test. A value of $p<0.05$ was considered to be statistically significant (see ESM Methods).

\section{Results}

Three month LND markedly reduced plasma nitrite/ nitrate levels The 3 month LND markedly reduced plasma nitrite/nitrate levels compared with the RD (Fig. 1a). To examine the underlying mechanism, we evaluated NOS levels in isolated aorta and visceral fat. eNOS protein levels in the aorta were comparable in the two diets (Fig. 1b), whereas, intriguingly, levels in EWAT were markedly lower with the LND than the RD (Fig. 1c), accounting for the markedly reduced plasma nitrite/nitrate levels induced by the LND. No nNOS or iNOS was found in EWAT with either diet (ESM Fig. 1a).

Three month LND resulted in metabolic syndrome-like conditions There were no significant differences in food intake (Fig. 1d), water intake (ESM Fig. 1b), or body weight (ESM Fig. 1c) between the 3 month LND and RD. However, the 3 month LND significantly increased EWAT weight (Fig. 1e), epididymal white adipocyte size (Fig. 1f) and plasma levels of total cholesterol (Fig. 2a), LDL-cholesterol (Fig. 2b) and small
Fig. 1 Effects of 1.5-4.5 months of the LND on plasma nitrite/ nitrate, eNOS levels, food intake and visceral fat in WT mice. (a) Plasma nitrite/nitrate levels $(n=8-12)$. (b, c) eNOS protein levels in the aorta $(\mathbf{b} ; n=6)$ and EWAT induced by 3 months of the LND (c; $n=10)$. (d, e) Food intake $(n=10-12)$ and relationship of EWAT to body weight $(n=10-12)$. (f) Epididymal white adipocyte size induced by 3 months of the LND $(n=10)$. Scale bars, $50 \mu \mathrm{m}$.

White bars, RD; black bars, LND. $* p<0.05, * * p<0.01$,

$* * * p<0.001$
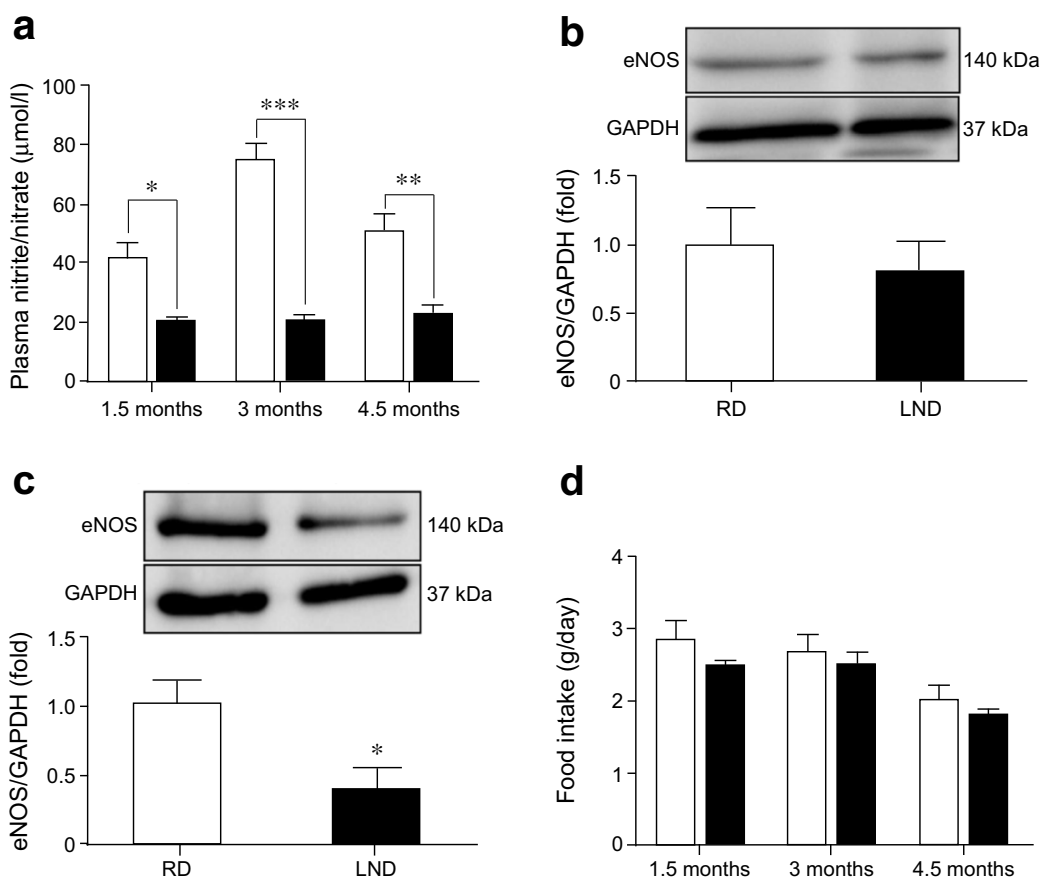

d

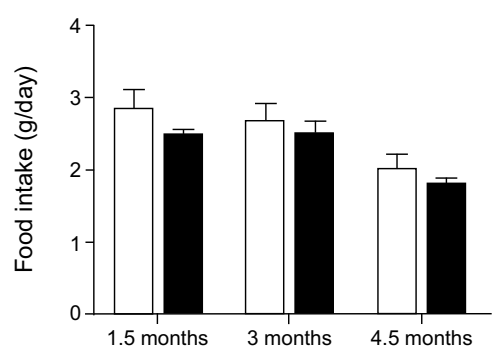

e

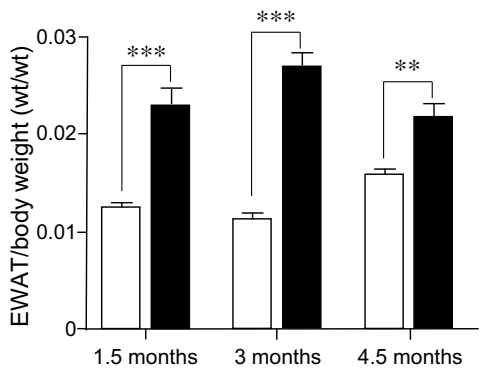

f

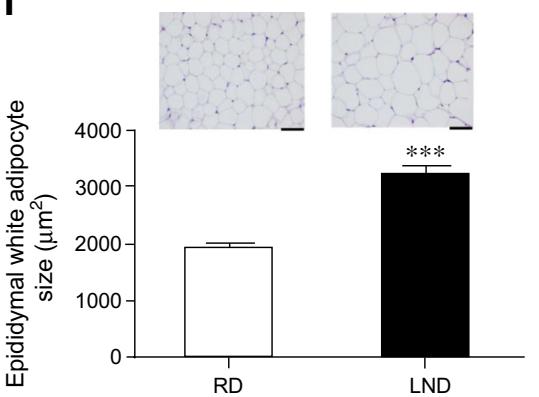


Fig. 2 Effects of 1.5-4.5 months of the LND on plasma lipid, blood glucose and insulin response. (a) Plasma total cholesterol levels ( $n=10-12)$. White bars, RD; black bars, LND. (b) Plasma LDL-cholesterol (LDL-c) levels induced by 3 months of the LND $(n=12)$. (c-e) Blood glucose levels after i.p. injection of $1 \mathrm{~g} / \mathrm{kg}$ glucose after 1.5 (c), 3 (d) and 4.5 (e) months of the LND $(n=11-12)$. (f-h) Percentage change in blood glucose levels after i.p. injection of $0.3 \mathrm{U} / \mathrm{kg}$ insulin after 1.5 (f), 3 (g) and 4.5 (h) months of the LND ( $n=8-12)$. White bars and white circles, RD; black bars and black squares, LND. $* p<0.05$, $* * p<0.01, * * * p<0.001$

\section{a}

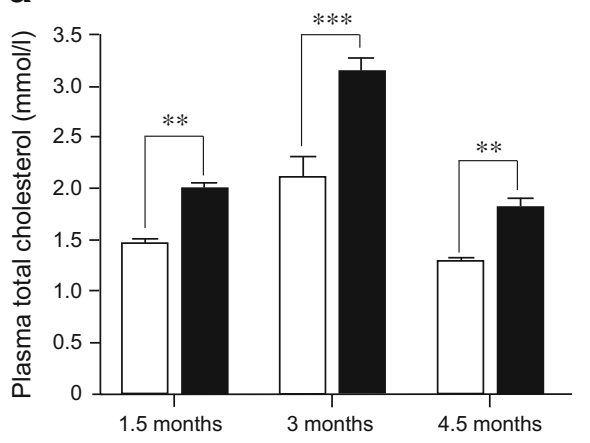

b
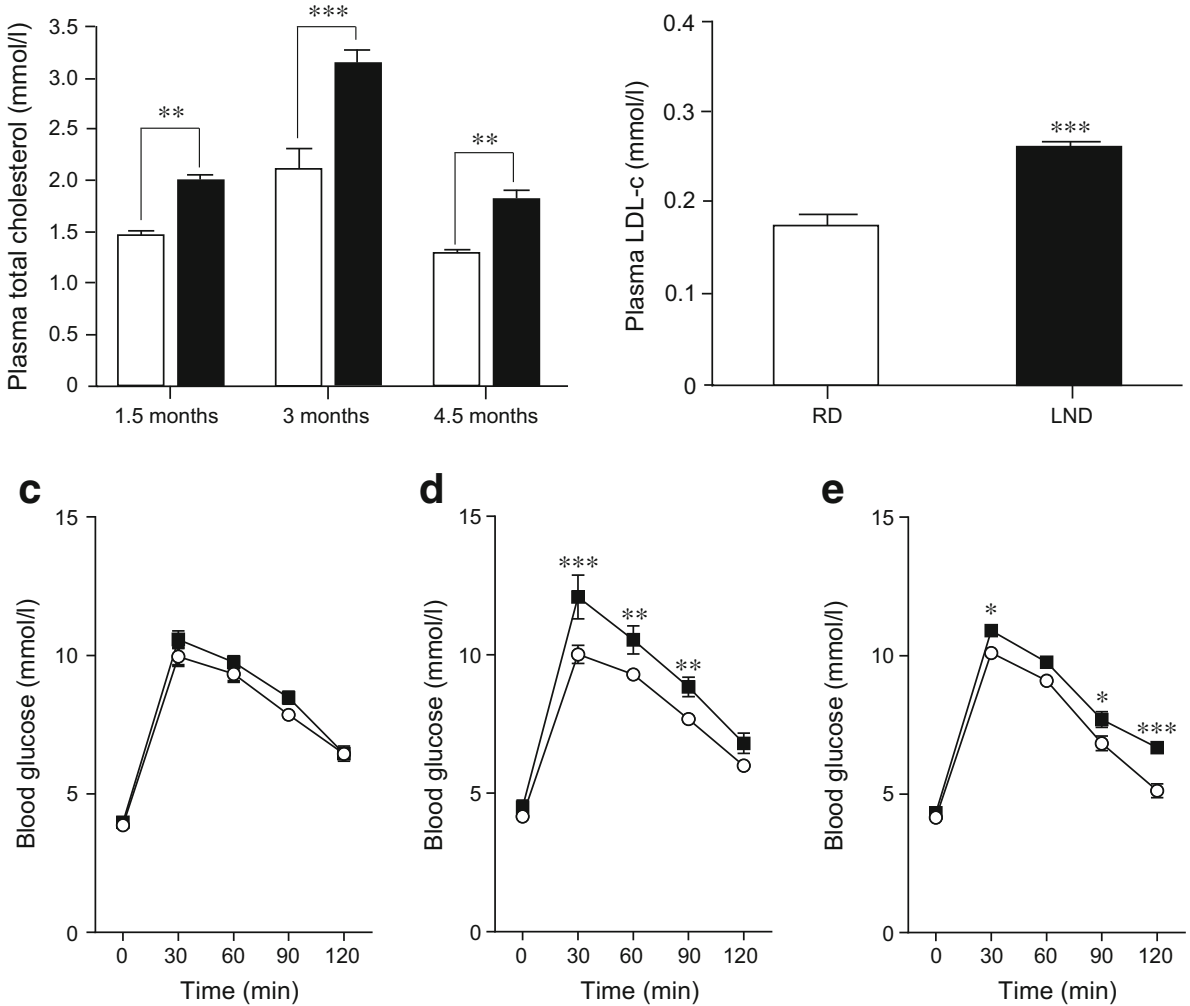

d

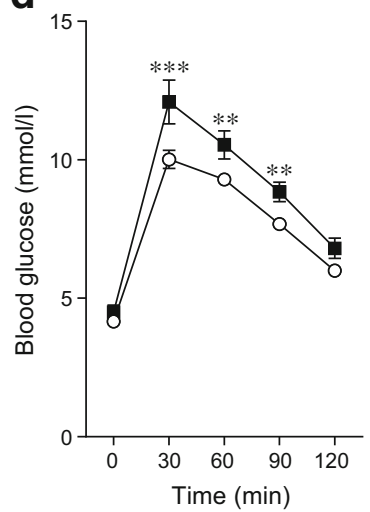

e

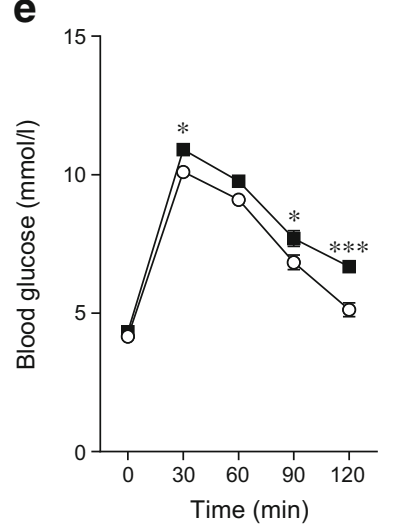

f

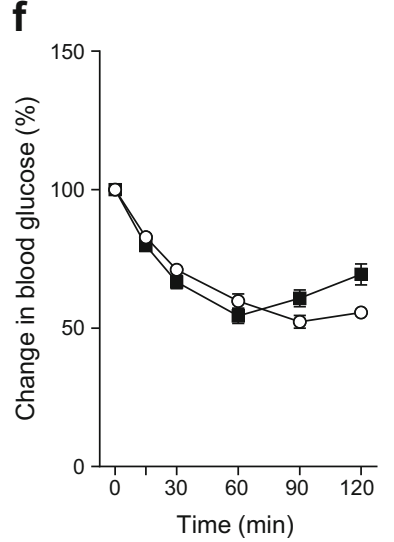

g

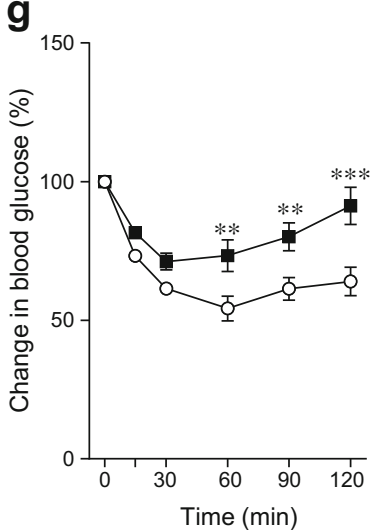

h

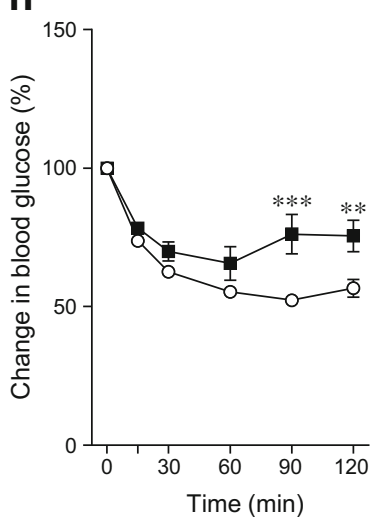

dense LDL-cholesterol (ESM Fig. 1d), and non-significantly elevated plasma triacylglycerol levels compared with the RD $(p=0.54)$ (Table 3). Furthermore, the 3 month LND significantly augmented blood glucose levels after i.p. glucose injection and aggravated blood glucose-lowering responses to i.p. insulin injection (Fig. 2d, g). Arterial blood pressure levels were similar with both diets (ESM Fig. 2a).

We next studied the time course of the metabolic effects at $1.5,3$ and 4.5 months after the start of the LND. The effects on EWAT weight (Fig. 1e), plasma total cholesterol levels (Fig. 2a), blood glucose levels after glucose injection (Fig. 2c-e) and blood glucose-lowering responses to insulin (Fig. 2f-h, ESM Fig. 1e-g) were time-dependent and appeared to reach a plateau at 3 months. There were no significant differences in fasting plasma insulin levels between the mice fed the LND or the RD at any of the time points (ESM Fig. 2b).

Table 3 Plasma triacylglycerol levels at 1.5, 3 and 4.5 months after the start of RD or LND

\begin{tabular}{llll}
\hline Diet & \multicolumn{3}{l}{ Plasma triacylglycerol levels $(\mathrm{mmol} / \mathrm{l})$} \\
\cline { 2 - 4 } & 1.5 months & 3 months & 4.5 months \\
\hline RD $(n=6-12)$ & $1.23 \pm 0.07$ & $0.94 \pm 0.05$ & $1.06 \pm 0.07$ \\
LND $(n=6-12)$ & $1.25 \pm 0.07$ & $1.41 \pm 0.19$ & $1.21 \pm 0.19$ \\
\hline
\end{tabular}

Statistical analysis was performed by two-way factorial ANOVA followed by Bonferroni's post hoc test for multiple comparisons, and no significant differences were seen 
Adiponectin levels in EWAT were markedly lower for the 3 month LND compared with the RD (Fig. 3a). The EWAT levels of PPAR- $\gamma$, total AMPK and p-AMPK, but not sirtuin 1 , were also markedly reduced after 1 week of the LND in comparison to the RD (Fig. 3b-e).

Simultaneous oral treatment with $2 \mathrm{mmol} / 1$ sodium nitrate for 3 months significantly reversed the reduced plasma nitrite/ nitrate levels induced by the LND (Fig. 4a). It also nonsignificantly improved the LND-induced gain in EWAT weight ( $p=0.06$ ) (Fig. 4b), and significantly ameliorated the epididymal white adipocyte hypertrophy, hyper-small dense LDL-cholesterolaemia, impaired glucose tolerance, reduced blood glucose-lowering responses to insulin, eNOS downregulation and adiponectin insufficiency induced by the LND (Fig. 4c-h, ESM Fig. 2c).

\section{Dysbiosis of gut microbiota was noted in the LND-fed mice} Although there was no significant difference between the two diets in the number of total sequencing reads (ESM Fig. 3a), there were significantly fewer operational taxonomic units, which represent the kind of gut bacteria, with the LND than the RD, suggesting less diversity of gut microbiota (Fig. 5a). Significantly different gut bacteria in each rank are shown in Fig. 5b, c and ESM Fig. 3b-j. In the phylum rank, there were more Actinobacteria with the LND than the RD (ESM Fig. 3b). In the class rank, there were more Actinobacteria and fewer Betaproteobacteria with the LND (ESM Fig. 3c, d). In the order rank, Bifidobacteriales was more numerous with the LND, and Burkholderiales and Bacillales were less numerous (ESM Fig. 3e, f). In the family (ESM Fig. 3g, h), the genus (Fig. 5b, c) and the species (ESM Fig. 3i, j) ranks, more than three gut bacteria were more prevalent, and more than two gut bacteria were less prevalent with the LND. On the other hand, Bacteroides fragilis was to a certain degree present with the $\mathrm{RD}$, but absent with the LND (median and interquartile range 312 [118-446] for the RD vs 0 for the LND; $n=5$ ). There were no significant differences between the two diets in Firmicutes, Bacteroidetes or Firmicutes/Bacteroidetes ratio, which might change in disease states of the metabolic syndrome [30-33], or Akkermansia muciniphila, which might improve the metabolic syndrome [34, 35] (data not shown). Bacteroides uniformis CECT 7771, which might improve the metabolic syndrome [36], was not detected in either diet.

An 18 month LND resulted in more severe metabolic syndrome and endothelial dysfunction Although the metabolic effects of the LND appeared to reach a plateau at 3 months, we explored the effects of an extremely long period of LND (18 months) for confirmation. There were no significant differences in food or water intake between the 18 month LND and RD (ESM Fig. 4a, b), but, notably, 18 months of the LND resulted in more severe metabolic syndrome, eliciting a significant gain in body weight, which was not seen until 4.5 months after the start of the LND (Fig. 6a). Micro-CT
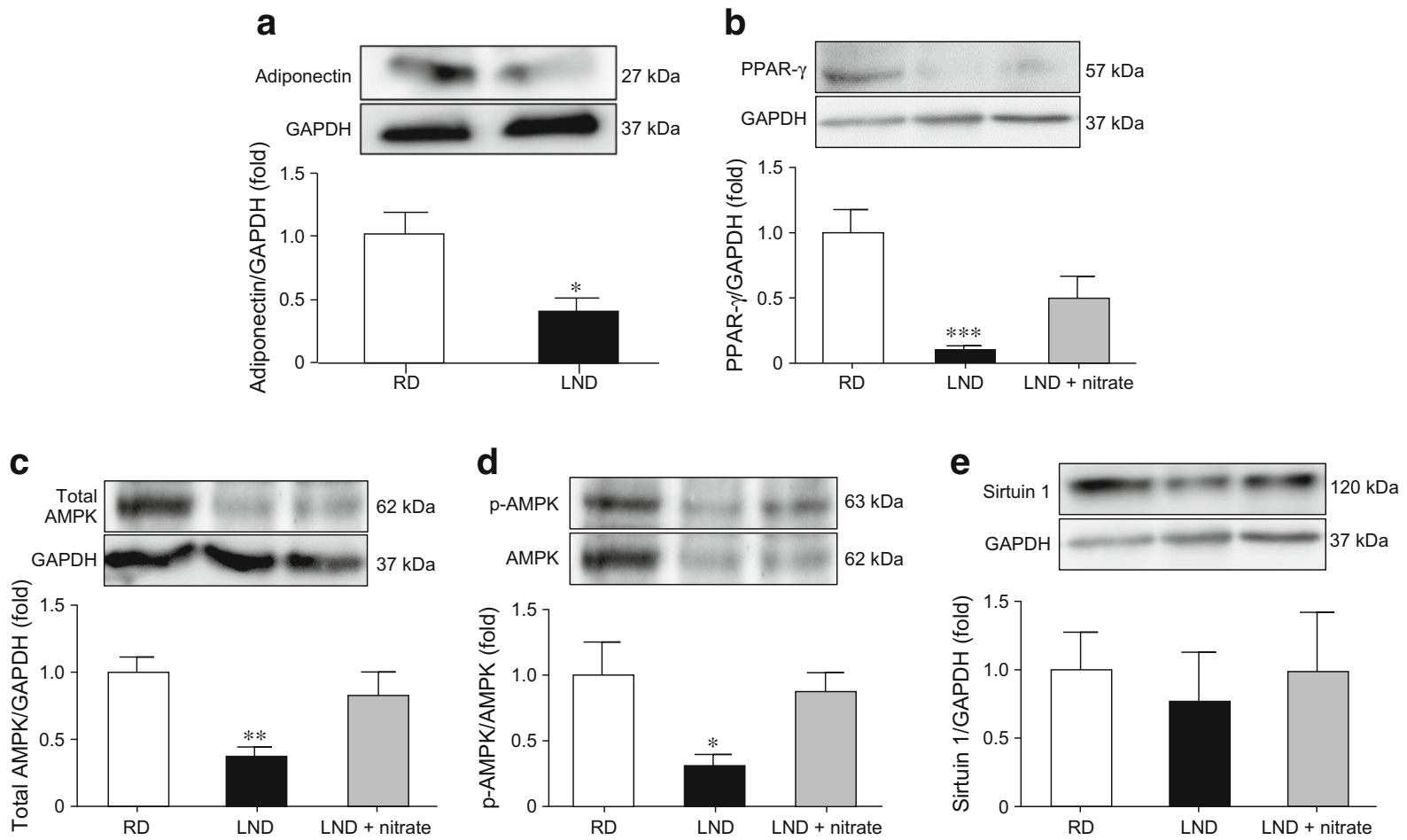

Fig. 3 Effects of 3 months of the LND and nitrate supplementation on adiponectin, and effects of 1 week of the LND and nitrate supplementation on PPAR- $\gamma$, total AMPK, p-AMPK and sirtuin 1 levels in EWAT. (a)

Adiponectin levels $(n=5)$. (b-e) PPAR- $\gamma$, total AMPK, p-AMPK and sirtuin 1 levels $(n=7)$. * $p<0.05, * * p<0.01, * * * p<0.001$ 
Fig. 4 Effects of 3 months of nitrate supplementation on metabolic syndrome-like conditions induced by the LND. (a) Plasma nitrite/nitrate levels ( $n=6-12$ ). (b) Relationship of EWAT to body weight $(n=7-8)$. (c) Epididymal white adipocyte size $(n=10)$. Scale bar, $50 \mu \mathrm{m}$. (d) Plasma small dense LDL-cholesterol (LDL-c) levels $(n=10-12)$. (e) Blood glucose levels after i.p. injection of $1 \mathrm{~g} / \mathrm{kg}$ glucose $(n=8)$. (f) Percentage change in blood glucose levels after i.p. injection of $0.3 \mathrm{U} / \mathrm{kg}$ insulin $(n=9-10)$. Black squares, LND; white triangles, LND + nitrate. (g, h) eNOS ( $n=5-6)$ and adiponectin $(n=3)$ levels in EWAT. $* p<0.05, * * p<0.01$, $* * * p<0.001$
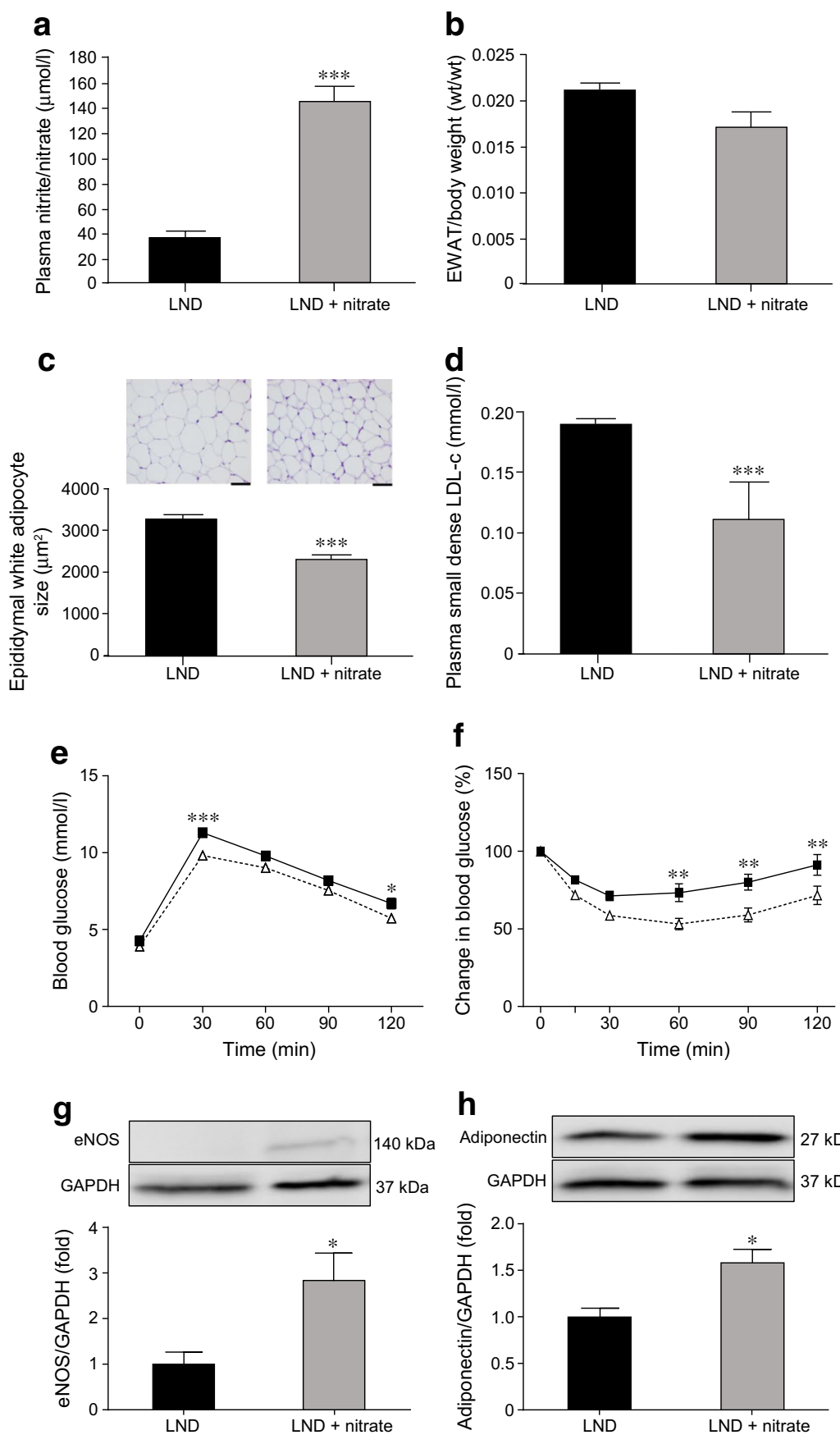

imaging indicated that body fat was markedly increased, by 3.2 times, in the 18 month LND-fed mice (Fig. 6b, c), and this was specifically due to an increase in visceral fat (Fig. 6d) and not subcutaneous fat (ESM Fig. 4c). The 18 month LND elevated levels of plasma small dense LDL-cholesterol and blood glucose following glucose injection, and significantly blunted blood glucose-lowering responses to insulin (Fig. 6e-g, ESM Fig. 4d). In addition, it significantly increased levels of fasting blood glucose, fasting plasma insulin and arterial blood pressure (Fig. $7 \mathrm{a}-\mathrm{c}$ ), findings that were not observed until 4.5 months after the start of the LND. eNOS protein levels in the EWAT were also significantly decreased with the 18 month LND (ESM Fig. 4e).

As the 18 month LND induced severe metabolic syndrome, we next examined vascular reactivity. In isolated aortas, contractions in response to phenylephrine, an adrenergic $\alpha_{1}$-receptor agonist, and endothelium-independent relaxations to diethylamine NONOate, an NO donor, were comparable between the two diets (ESM Fig. 4f, g), whereas endotheliumdependent relaxation in response to acetylcholine, a physiological eNOS activator, was significantly impaired with the LND (Fig. 7d). eNOS protein levels were significantly diminished in 
Fig. 5 Effects of 3 months of the LND on gut microbiota. (a) Operational taxonomic units $(n=5$ each). (b, c) Significantly higher (b) or lower (c) numbers of gut bacteria in the genus rank $(p<0.05, n=5$ each). White boxes, RD; grey boxes, LND. Insets are the magnified views of sequencing reads of the rightmost three gut bacteria in parts $\mathbf{b}$ and $\mathbf{c}$. $* p<0.05$
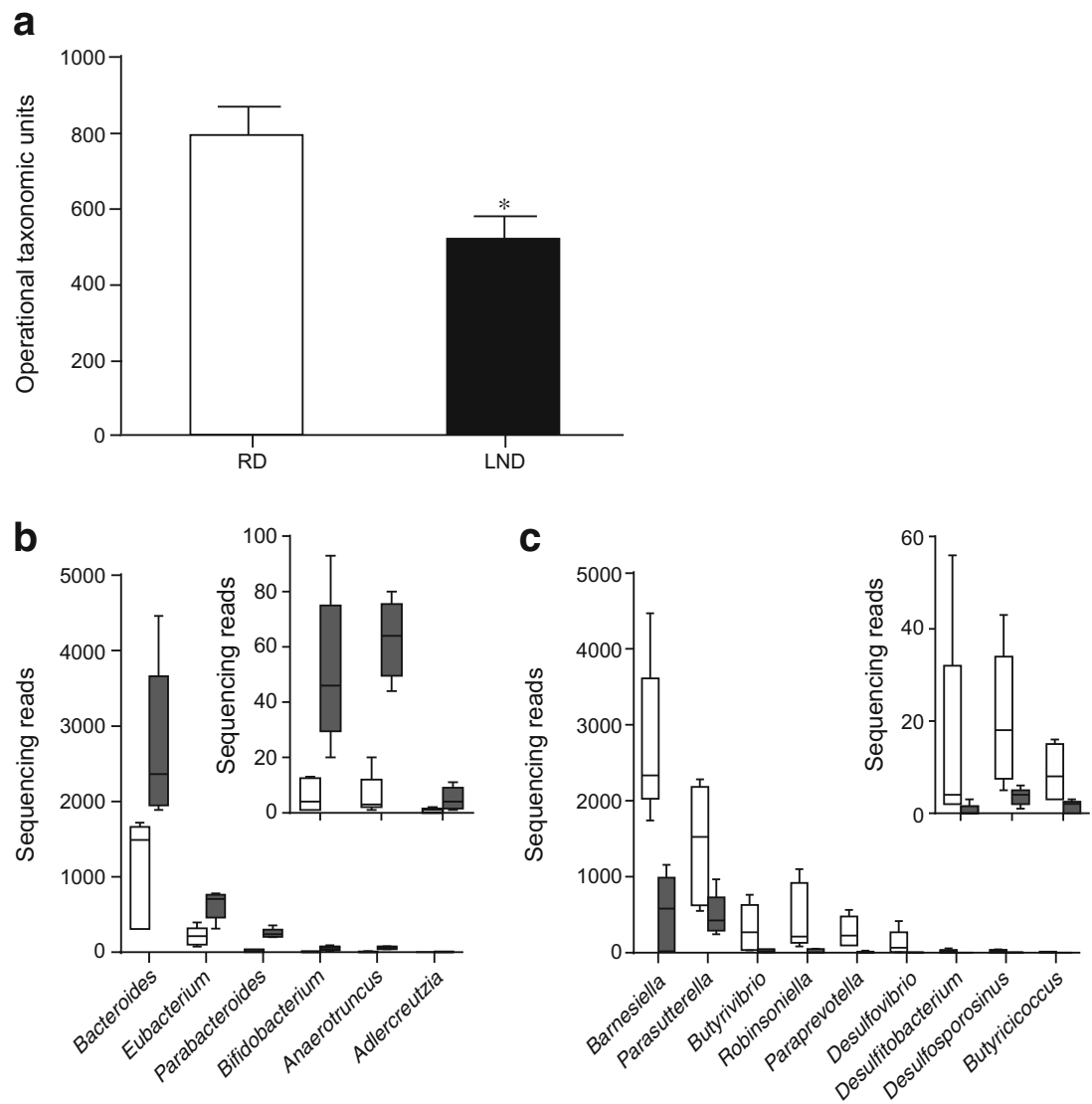

the aortas (Fig. 7e), and there was a non-significant tendency with the LND towards lower levels of phosphorylation of eNOS at serine $1177(p=0.12)$, which is an index of eNOS activation, without affecting the phosphorylation levels of eNOS at threonine 495, which is an index of eNOS inactivation (ESM Fig. 4h, i).

Simultaneous treatment with sodium nitrate for 18 months inhibited these metabolic abnormalities, the endothelial dysfunction and the aortic and EWAT eNOS downregulation induced by the LND (Figs 6, 7a-e, ESM Fig. 4c-e).

There were no significant increases in the serum levels of 23 inflammatory markers measured using the Bio-Plex system between the 1.5 month LND and RD (Table 4). There were also no significant differences in the number of inflammatory foci in the EWAT and peri-renal WAT between the 3 month LND and RD (data not shown). However, the 18 month LND non-significantly increased the number of inflammatory foci in the EWAT $(p=0.08)$ and significantly augmented those in the peri-renal WAT compared with the RD (Fig. 7f, g).

A 22 month LND led to cardiovascular death We experienced sudden death in some of the LND-fed mice. During the 22 months of follow-up, none (0/24) of the RD-fed mice, but $31.8 \%$ (7/22) of the LND-fed mice, died. The survival rate was significantly worse in the LND-fed than in the RD-fed mice, and co-treatment with sodium nitrate improved the reduced survival (Fig. 8a). We performed a post mortem histopathological analysis to identify the cause of death in the seven dead LND-fed mice. We were not able to investigate the cause of death in one mouse because of strong post mortem putrefaction. We judged that one mouse had died of an acute anterior wall myocardial infarction (Fig. 8b), and that one mouse died of malignant lymphoma of the lung, liver (Fig. 8g), kidney and spleen. In the other four mice, we noted coronary perivascular fibrosis, pulmonary congestion and acute renal tubular necrosis (Fig. 8d-f), findings that are observed in cardiac sudden death. Myocardial fibrosis, which might have resulted from myocardial infarction, was seen in one mouse (Fig. 8c), malignant lymphoma of the liver and spleen was observed in one mouse, and no other pathological findings that could explain the cause of death were seen in any of the mice.

\section{Discussion}

In previous studies, the effects of an LND on cardiac and liver ischaemia-reperfusion injury [21-23], cardiac allograft rejection [23] and platelet aggregation [24] were investigated using a commercially available low-nitrite/nitrate chow, but the contents of L-arginine, fat, carbohydrates, protein and energy were 
a

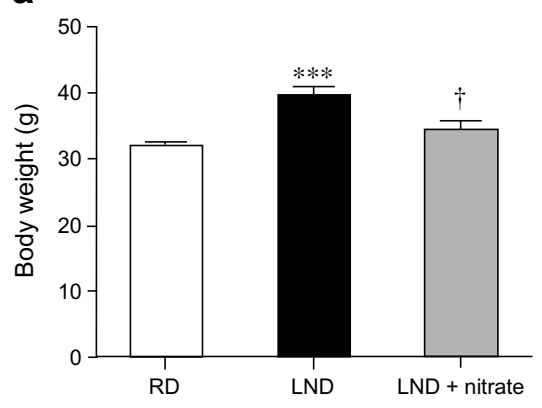

C

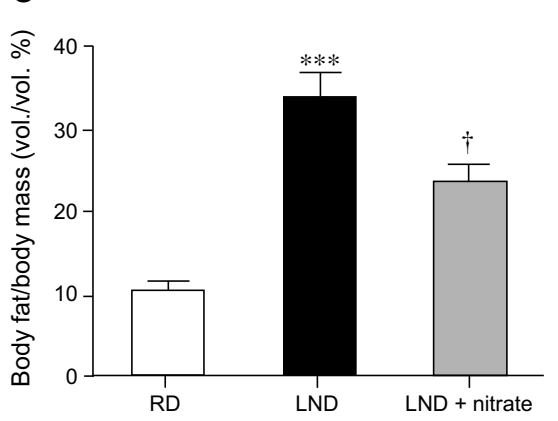

b

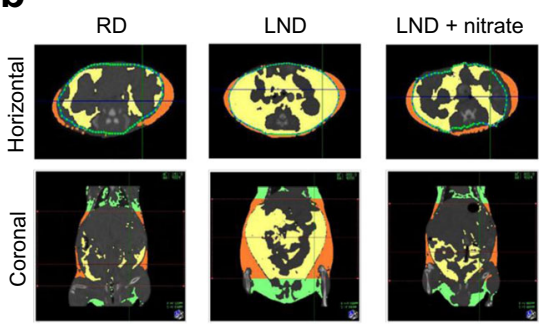

d

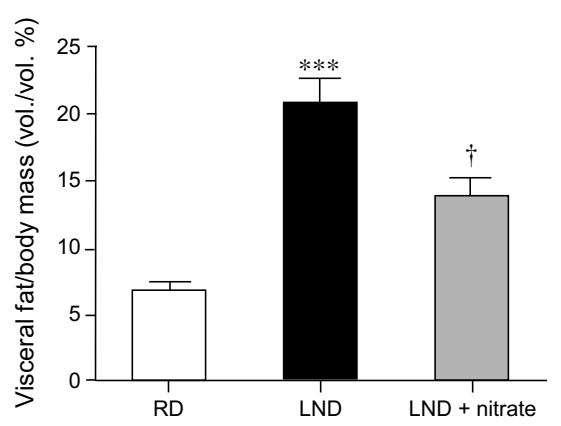

e

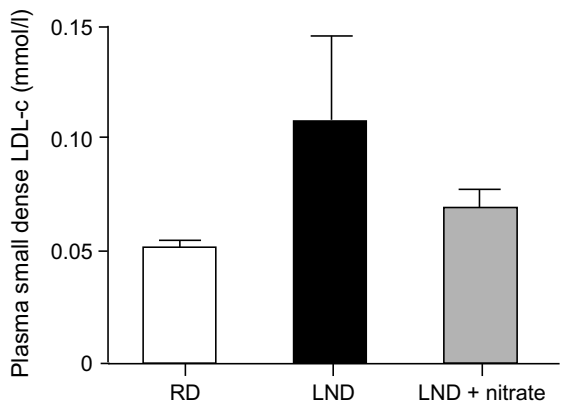

f

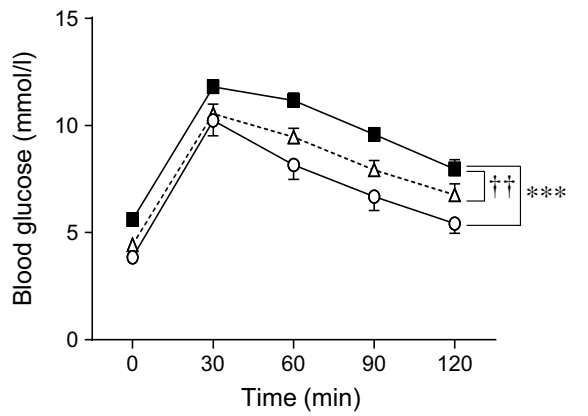

g

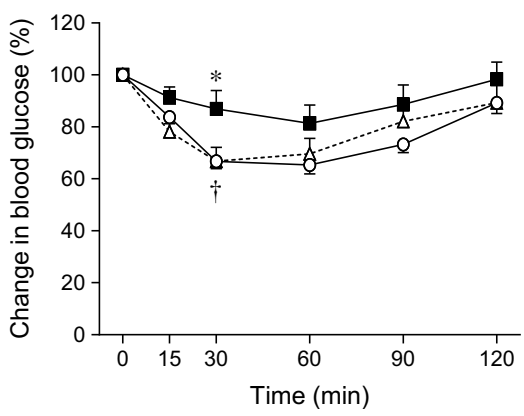

Fig. 6 Effects of 18 months of the LND and nitrate supplementation on body weight, body fat, plasma small dense LDL-cholesterol, blood glucose and insulin sensitivity. (a) Body weight $(n=12-16)$. (b) Micro-CT images. Yellow, visceral fat; orange, subcutaneous fat. (c) Relationship of body fat to body mass $(n=11-12)$. (d) Relationship of visceral fat to body mass $(n=11-12)$. (e) Levels of plasma small dense LDL-cholesterol
(LDL-c; $n=7-9$ ). (f) Blood glucose levels after i.p. injection of $1 \mathrm{~g} / \mathrm{kg}$ glucose $(n=12-14)$. (g) Percentage change in blood glucose levels after i.p. injection of $0.3 \mathrm{U} / \mathrm{kg}$ insulin $(n=10)$. White circles, RD; black squares, LND; white triangles, LND + nitrate. $* p<0.05$, *** $p<0.001$ for RD vs LND; ${ }^{\dagger} p<0.05,{ }^{\dagger \dagger} p<0.01$ for LND vs LND + nitrate

production system to that regulation might be minor. However, contrary to these suggestions, another study showed that a 1 week LND, compared with an RD, markedly decreased plasma nitrite/nitrate levels in WT mice showing normal NOS activities [21]; however, the possible underlying mechanisms remain to be clarified.

In this study, we obtained a similar finding in that 1.5-4.5 months of the LND markedly reduced plasma nitrite/ nitrate levels in WT mice. We then examined the causative mechanisms, and found that eNOS levels in the visceral fat, but not in the aorta, were markedly suppressed in the 3 month LND-fed mice, accounting for the markedly depressed plasma 

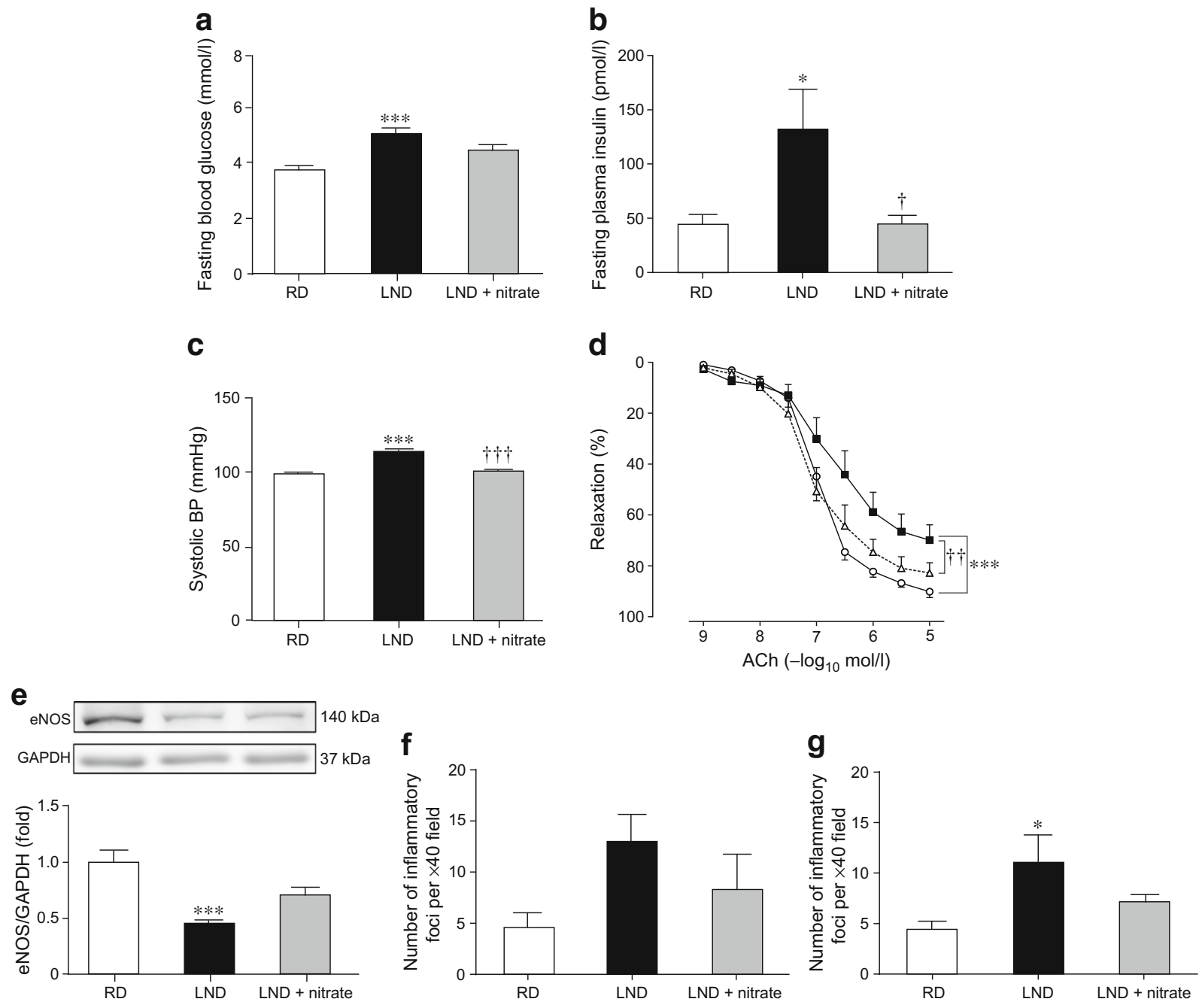

Fig. 7 Effects of 18 months of the LND and nitrate supplementation on fasting blood glucose, blood pressure, vascular reactivity, eNOS levels and inflammation. (a) Fasting blood glucose levels $(n=12-14)$. (b) Fasting plasma insulin levels $(n=6-7)$. (c) Systolic BP $(n=12-15)$. (d) Endothelium-dependent relaxation in response to acetylcholine

(ACh) $(n=6)$. White circles, RD; black squares, LND; white triangles, LND + nitrate. (e) eNOS protein levels in the aorta $(n=6)$. (f, g) Number of inflammatory foci in EWAT (f) and peri-renal WAT $(\mathbf{g})(n=12-14)$. $* p<0.05, * * * p<0.001$ for RD vs LND; ${ }^{\dagger} p<0.05,{ }^{\dagger \dagger} p<0.01$, ${ }^{\dagger \dagger} p<0.001$ for LND vs LND + nitrate

nitrite/nitrate levels induced by the LND. We also found that adiponectin levels in the visceral fat were remarkably low in the 3 month LND-fed mice. Adiponectin has been reported to upregulate eNOS [39], so the adiponectin insufficiency may have mediated the visceral adipose eNOS downregulation induced by the LND. It has also been reported that eNOS enhances adiponectin levels in adipocytes [40]. Thus, there may be a vicious cycle of adiponectin insufficiency and eNOS downregulation, and this vicious cycle may contribute to the markedly diminished plasma nitrite/nitrate levels induced by the LND.

The levels of PPAR- $\gamma$, total AMPK and p-AMPK were significantly lower in the EWAT in the 1 week LND-fed mice compared with the RD-fed mice. As it has been reported that PPAR- $\gamma$ and AMPK increase levels of adiponectin levels in adipocytes [41, 42], it is conceivable that reduced PPAR- $\gamma$ and AMPK in the visceral fat were involved in the adiponectin insufficiency induced by the LND.

The 3 month LND-fed mice exhibited visceral obesity with adipocyte hypertrophy, hyper-LDL-cholesterolaemia and hyper-small dense LDL-cholesterolaemia and glucose intolerance, and the 18 month LND-fed mice manifested body weight gain, hypertension, insulin resistance and endothelial dysfunction. These changes eventually resulted in death due to cardiovascular disease, including acute myocardial infarction. These abnormalities were reversed by concurrent nitrate supplementation, indicating that the observed effects were indeed caused by dietary nitrite/nitrate deficiency. It is thus evident that long-term dietary nitrite/nitrate deficiency can cause the metabolic syndrome, endothelial dysfunction and cardiovascular death in mice. The 3 and 4.5 month LND significantly reduced blood glucose-lowering responses to insulin, but did not significantly affect fasting plasma insulin levels. Thus, before 18 months, the effects of LND on glucose tolerance could be due to the result of those on pancreatic beta cell function. 
Table 4 Serum levels of 23 inflammatory markers in mice fed the RD and LND for 1.5 months

\begin{tabular}{|c|c|c|c|}
\hline \multirow[t]{2}{*}{ Marker } & \multicolumn{2}{|c|}{ Serum levels $(\mathrm{pg} / \mathrm{ml})$} & \multirow[t]{2}{*}{$p$ value } \\
\hline & $\mathrm{RD}$ & LND & \\
\hline $\mathrm{IL}-1 \alpha$ & $31.3 \pm 4.5$ & $28.0 \pm 3.0$ & 0.55 \\
\hline IL-1 $\beta$ & $643.9 \pm 67.2$ & $558.5 \pm 72.1$ & 0.40 \\
\hline IL-2 & $110.4 \pm 18.2$ & $93.4 \pm 19.2$ & 0.54 \\
\hline IL-3 & $27.5 \pm 4.6$ & $23.5 \pm 4.2$ & 0.53 \\
\hline IL-4 & $51.7 \pm 8.3$ & $46.6 \pm 9.3$ & 0.69 \\
\hline IL-5 & $89.5 \pm 14.2$ & $72.8 \pm 13.1$ & 0.40 \\
\hline IL-6 & $67.3 \pm 8.6$ & $55.7 \pm 8.3$ & 0.34 \\
\hline IL-9 & $580.2 \pm 54.8$ & $544.4 \pm 33.7$ & 0.60 \\
\hline IL-10 & $217.9 \pm 56.5$ & $134.3 \pm 23.4$ & 0.19 \\
\hline IL-12p40 & $459.1 \pm 26.7$ & $401.8 \pm 31.3$ & 0.18 \\
\hline IL-12p70 & $1084.5 \pm 118.6$ & $800.0 \pm 139.3$ & 0.14 \\
\hline IL-13 & $724.3 \pm 104.6$ & $714.4 \pm 108.3$ & 0.95 \\
\hline IL-17 & $342.7 \pm 30.0$ & $275.5 \pm 30.2$ & 0.13 \\
\hline TNF- $\alpha$ & $847.6 \pm 103.5$ & $632.7 \pm 107.4$ & 0.17 \\
\hline $\mathrm{IFN}-\gamma$ & $111.6 \pm 14.5$ & $85.7 \pm 15.7$ & 0.24 \\
\hline MCP-1 & $746.2 \pm 72.7$ & $590.0 \pm 70.2$ & 0.14 \\
\hline MIP-1 $\alpha$ & $74.4 \pm 8.4$ & $59.0 \pm 10.0$ & 0.26 \\
\hline MIP-1 $\beta$ & $131.6 \pm 19.0$ & $105.1 \pm 16.8$ & 0.31 \\
\hline G-CSF & $184.7 \pm 22.4$ & $128.6 \pm 18.2$ & 0.07 \\
\hline GM-CSF & $456.9 \pm 19.2$ & $437.8 \pm 25.7$ & 0.56 \\
\hline $\mathrm{KC}$ & $137.6 \pm 16.9$ & $118.7 \pm 8.1$ & 0.33 \\
\hline Eotaxin & $1459.7 \pm 75.0$ & $1397.2 \pm 104.0$ & 0.63 \\
\hline RANTES & $34.2 \pm 2.2$ & $23.4 \pm 1.3$ & 0.0004 \\
\hline
\end{tabular}

$n=8-10$ mice for each group

G-CSF, granulocyte-colony stimulating factor; GM-CSF, granulocyte/ macrophage-colony stimulating factor; $\mathrm{KC}$, keratinocyte-derived chemokine; MCP, monocyte chemotactic protein; MIP, macrophage inflammatory protein; RANTES, regulated on activation normal cell expressed and secreted

A clustering of cardiovascular risk factors (i.e. the metabolic syndrome) could have contributed to the development of endothelial dysfunction and cardiovascular death in the LNDfed mice. On the other hand, hyper-LDL-cholesterolaemia and hyper-small dense LDL-cholesterolaemia, both of which are independent cardiovascular risk factors [43], were also noted in the LND-fed mice. The small dense LDL-cholesterol particle can easily penetrate the vascular wall because of its small particle size, and is related more strongly to the risk of cardiovascular disease. It is thus likely that those factors may also have been independently involved in the occurrence of endothelial dysfunction and cardiovascular death in the LND-fed mice.

The 18 month LND-fed mice displayed an impairment of endothelium-dependent relaxation in response to acetylcholine, a physiological eNOS activator, along with aortic eNOS downregulation, suggesting the presence of coronary vasospasm. Coronary vasospasm-elicited myocardial ischaemia can lead to fatal cardiac arrhythmia and/or cardiogenic shock. On the other hand, the dead LND-fed mice showed acute myocardial infarction, myocardial fibrosis that might have resulted from myocardial infarction and coronary perivascular fibrosis. They also exhibited pulmonary congestion and acute renal tubular necrosis, both of which are seen in sudden cardiac death. Taking these findings together, we thought that $83.3 \%(5 / 6)$ of the dead LND-fed mice showed changes consistent with cardiovascular death.

The data obtained at 3 and 4.5 months are the most important and significant, as the changes in glucose metabolism, lipid biology, body fat distribution and gut microbiome occurred independent of food intake and body weight. On the other hand, the morbidity and mortality experiments at 18 and 22 months, respectively, are complicated by greater weight gain in the LND group, making it difficult to separate the effects of obesity from those of the nitrite/nitrate deficiency.

We conducted the glucose and insulin tolerance tests under anaesthesia. Whereas it has been reported that high-fat dietfed C57BL/6J mice showed glucose intolerance compared with their RD-fed counterparts whether they were tested under conscious or anesthetised conditions [44], it has also been indicated that the use of anaesthesia could influence glucose tolerance in C57BL/6J mice [45]. Therefore, the use of anaesthesia could be a limitation of this study.

An increased number of inflammatory foci, decreased eNOS levels and lower adiponectin levels were noted in the visceral fat of the LND-fed mice, and improvements in the LND-induced metabolic syndrome by nitrate supplementation were linked to ameliorations of these changes. As it has been reported that inflammation, eNOS downregulation and adiponectin insufficiency contribute to the occurrence and progression of the metabolic syndrome [46-48], it is possible that those factors were involved in the development of the metabolic syndrome induced by the LND.

The following lines of evidence suggest a causal role of dysbiosis of the gut microbiota in the development of the metabolic syndrome. First, it has been reported that the composition of gut microbiota differs largely between lean individuals and patients with the metabolic syndrome [33]. Second, it has been indicated that transplantation of gut microbiota from obese humans with metabolic abnormalities into germ-free mice results in the development of obesity and metabolic abnormalities in the mice [49]. Third, it has been shown that the transfer of gut microbiota from lean healthy human participants into individuals with the metabolic syndrome improves insulin resistance in the latter group [33]. In our study, there were significantly fewer operational taxonomic units with the LND than with the RD, suggesting less diversity of gut microbiota in the LND-fed mice. There also were significantly different constituents of gut microbiota in a variety of the hierarchy ranks with the LND and the RD. In 
Fig. 8 Effects of 22 months of the LND and nitrate

supplementation on survival rate and post mortem findings in LND-fed mice. (a) Survival rate ( $n=15-24)$. Grey line, RD; black line, LND; dotted line, LND + nitrate. $* * p<0.01$. (b) Acute myocardial infarction. (c) Myocardial fibrosis. (d) Coronary perivascular fibrosis. (e) Pulmonary congestion. (f) Acute renal tubular necrosis. (g)

Malignant lymphoma. Scale bars, $100 \mu \mathrm{m}$

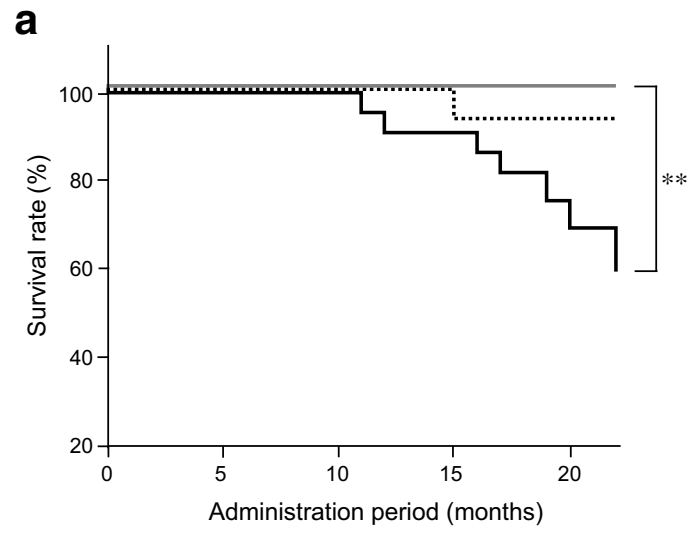

b

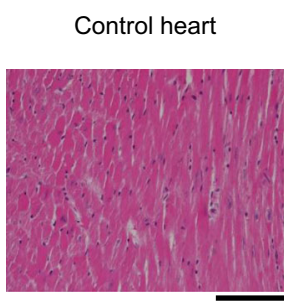

Acute myocardial infarction

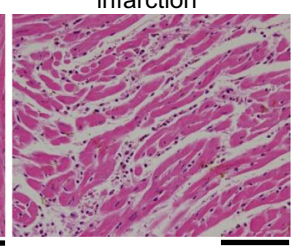

C

Control heart

Myocardial fibrosis
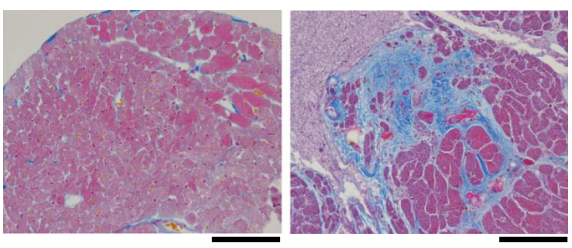

d

Control coronary artery

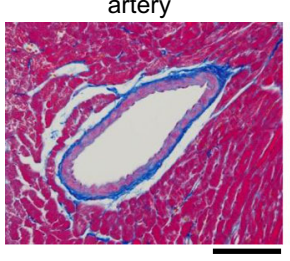

Coronary perivascular fibrosis

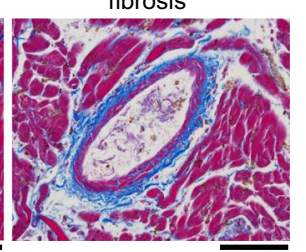

f

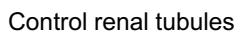

Acute renal tubular necrosis

e

Control lung

Pulmonary congestion
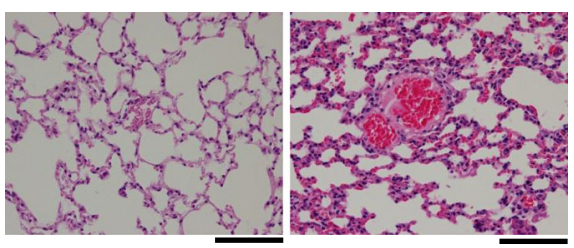

g

Malignant lymphoma in lung

Malignant lymphoma

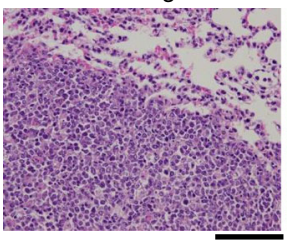
in liver
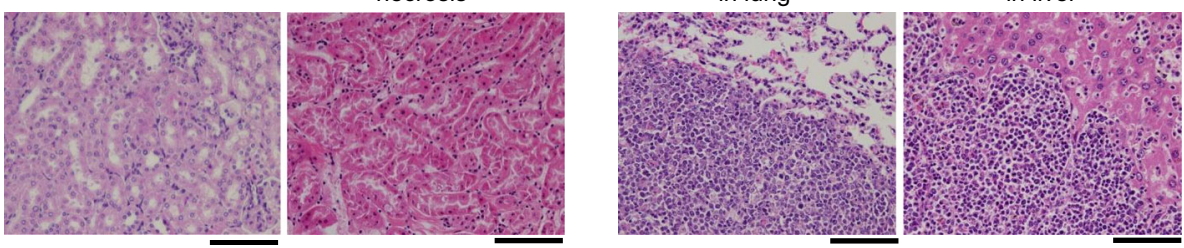

agreement with these results, Vrieze et al. indicated that less diversity and fewer distinct constituents of gut microbiota were recognised in individuals with the metabolic syndrome, and that the improvement of insulin resistance after transfer of the gut microbiota was accompanied by an amelioration of the reduced diversity and distinct constituents of gut microbiota [33]. It is thus possible that dysbiotic gut microbiota were involved in the pathogenesis of the metabolic syndrome induced by the LND.

We completely matched the energy contents in the two diets, and food consumption was comparable between the LND and RD at 1.5, 3, 4.5 and 18 months after the start of

the diet, suggesting a similar energy intake in the LND and RD. Nevertheless, the LND-fed mice developed the metabolic syndrome. Therefore, we may have succeeded for the first time in identifying specific dietary ingredients that cause the metabolic syndrome even in the absence of excessive intake of energy.

In summary, we were able to demonstrate that long-term dietary nitrite/nitrate deficiency gave rise to the metabolic syndrome, endothelial dysfunction and eventually cardiovascular death in mice, indicating a novel pathogenetic role of the exogenous NO production system in the metabolic syndrome and its vascular complications. 
Funding This work was supported in part by Grant-in-Aids for Research Activity Start-up (15H06519) and Scientific Research (C) (16K09519) from the Japan Society for the Promotion of Science, Special Account Budgets for Education and Research granted by the Japan Ministry of Education, and Grants from the Okinawa Medical Science Research Foundation, the Ryukyu University Support Foundation, the Promotion Project of Medical Clustering of Okinawa Prefecture, the Okinawa Prefecture for Promotion of Advanced Medicine and the University of the Ryukyus, Japan.

Duality of interest The authors declare that there is no duality of interest associated with this manuscript.

Data availability The datasets generated during and/or analysed during the current study are available from the corresponding author on reasonable request.

Contribution statement MK-T. and MS designed the study, acquired, analysed and interpreted data, and drafted the article. AT acquired, analysed and interpreted data, and drafted the article. TK, TM, KN, TU, JN, CK, MI, HK, YTa, Yto, S-iK, JO and KS acquired, analysed, and interpreted data. HSu and HM designed the study, analysed and interpreted data. HSh, NY, SM, YO, MM and AA analysed and interpreted data. MT designed the study, analysed and interpreted data, drafted the article. All authors critically revised the article for important intellectual content and gave final approval of the version to be published. MT is the guarantor of this work.

\section{References}

1. Eckel R (2015) The Metabolic Syndrome. In: Kasper DL, Fauci AS, Hauser SL, Longo DL, Jameson JL, Loscaldo J (eds) Harrison's Principles of Internal Medicine. Mc Graw Hill Education, New York, pp 2449-2454

2. Beltran-Sanchez H, Harhay MO, Harhay MM, McElligott S (2013) Prevalence and trends of metabolic syndrome in the adult U.S. population, 1999-2010. J Am Coll Cardiol 62:697-703

3. Alberti KG, Eckel RH, Grundy SM et al (2009) Harmonizing the metabolic syndrome: a joint interim statement of the International Diabetes Federation Task Force on Epidemiology and Prevention; National Heart, Lung, and Blood Institute; American Heart Association; World Heart Federation; International Atherosclerosis Society; and International Association for the Study of Obesity. Circulation 120:1640-1645

4. Mottillo S, Filion KB, Genest J et al (2010) The metabolic syndrome and cardiovascular risk a systematic review and meta-analysis. J Am Coll Cardiol 56:1113-1132

5. Chen J, Muntner P, Hamm LL et al (2004) The metabolic syndrome and chronic kidney disease in U.S. adults. Ann Intern Med 140: 167-174

6. Esposito K, Chiodini P, Colao A, Lenzi A, Giugliano D (2012) Metabolic syndrome and risk of cancer: a systematic review and meta-analysis. Diabetes Care 35:2402-2411

7. Garg PK, Biggs ML, Carnethon M et al (2014) Metabolic syndrome and risk of incident peripheral artery disease: the cardiovascular health study. Hypertension 63:413-419

8. Hamaguchi M, Kojima T, Takeda N et al (2005) The metabolic syndrome as a predictor of nonalcoholic fatty liver disease. Ann Intern Med 143:722-728

9. Hanley AJ, Karter AJ, Williams K et al (2005) Prediction of type 2 diabetes mellitus with alternative definitions of the metabolic syndrome: the Insulin Resistance Atherosclerosis Study. Circulation 112:3713-3721
10. Ignarro LJ (1990) Biosynthesis and metabolism of endotheliumderived nitric oxide. Annu Rev Pharmacol Toxicol 30:535-560

11. Moncada S, Higgs A (1993) The L-arginine-nitric oxide pathway. N Engl J Med 329:2002-2012

12. Murad F (2006) Shattuck Lecture. Nitric oxide and cyclic GMP in cell signaling and drug development. N Engl J Med 355:2003-2011

13. Tsutsui M, Shimokawa H, Otsuji Y, Ueta Y, Sasaguri Y, Yanagihara N (2009) Nitric oxide synthases and cardiovascular diseases: insights from genetically modified mice. Circ J 73:986-993

14. Tsutsui M, Shimokawa H, Otsuji Y, Yanagihara N (2010) Pathophysiological relevance of NO signaling in the cardiovascular system: novel insight from mice lacking all NO synthases. Pharmacol Ther 128:499-508

15. Tsutsui M, Tanimoto A, Tamura M et al (2015) Significance of nitric oxide synthases: Lessons from triple nitric oxide synthases null mice. J Pharmacol Sci 127:42-52

16. Kevil CG, Lefer DJ (2011) Review focus on inorganic nitrite and nitrate in cardiovascular health and disease. Cardiovasc Res 89: 489-491

17. Lundberg JO, Weitzberg E, Gladwin MT (2008) The nitrate-nitritenitric oxide pathway in physiology and therapeutics. Nat Rev Drug Discov 7:156-167

18. Omar SA, Webb AJ (2014) Nitrite reduction and cardiovascular protection. J Mol Cell Cardiol 73:57-69

19. Milkowski A, Garg HK, Coughlin JR, Bryan NS (2010) Nutritional epidemiology in the context of nitric oxide biology: a risk-benefit evaluation for dietary nitrite and nitrate. Nitric Oxide 22:110-119

20. Weitzberg E, Lundberg JO (2013) Novel aspects of dietary nitrate and human health. Annu Rev Nutr 33:129-159

21. Bryan NS, Calvert JW, Elrod JW, Gundewar S, Ji SY, Lefer DJ (2007) Dietary nitrite supplementation protects against myocardial ischemia-reperfusion injury. Proc Natl Acad Sci U S A 104:19144 19149

22. Bryan NS, Calvert JW, Gundewar S, Lefer DJ (2008) Dietary nitrite restores $\mathrm{NO}$ homeostasis and is cardioprotective in endothelial nitric oxide synthase-deficient mice. Free Radic Biol Med 45:468-474

23. Zhan J, Nakao A, Sugimoto R et al (2009) Orally administered nitrite attenuates cardiac allograft rejection in rats. Surgery 146 : 155-165

24. Park JW, Piknova B, Huang PL, Noguchi CT, Schechter AN (2013) Effect of blood nitrite and nitrate levels on murine platelet function. PLoS One 8:e55699

25. Usui S, Hara Y, Hosaki S, Okazaki M (2002) A new on-line dual enzymatic method for simultaneous quantification of cholesterol and triglycerides in lipoproteins by HPLC. J Lipid Res 43:805-814

26. Uchida T, Furuno Y, Tanimoto A et al (2014) Development of an experimentally useful model of acute myocardial infarction: $2 / 3$ nephrectomized triple nitric oxide synthases-deficient mouse. J Mol Cell Cardiol 77:29-41

27. Satoh K, Satoh T, Kikuchi N et al (2014) Basigin mediates pulmonary hypertension by promoting inflammation and vascular smooth muscle cell proliferation. Circ Res 115:738-750

28. Kim SW, Suda W, Kim S et al (2013) Robustness of gut microbiota of healthy adults in response to probiotic intervention revealed by high-throughput pyrosequencing. DNA Res 20:241-253

29. Qin N, Yang F, Li A et al (2014) Alterations of the human gut microbiome in liver cirrhosis. Nature 513:59-64

30. Lecomte V, Kaakoush NO, Maloney CA et al (2015) Changes in gut microbiota in rats fed a high fat diet correlate with obesityassociated metabolic parameters. PLoS One 10:e0126931

31. Ley RE, Backhed F, Turnbaugh P, Lozupone CA, Knight RD, Gordon JI (2005) Obesity alters gut microbial ecology. Proc Natl Acad Sci U S A 102:11070-11075

32. Turnbaugh PJ, Ley RE, Mahowald MA, Magrini V, Mardis ER, Gordon JI (2006) An obesity-associated gut microbiome with increased capacity for energy harvest. Nature 444:1027-1031 
33. Vrieze A, Van Nood E, Holleman F et al (2012) Transfer of intestinal microbiota from lean donors increases insulin sensitivity in individuals with metabolic syndrome. Gastroenterology 143:913916.e917

34. Everard A, Belzer C, Geurts L et al (2013) Cross-talk between Akkermansia muciniphila and intestinal epithelium controls dietinduced obesity. Proc Natl Acad Sci U S A 110:9066-9071

35. Shin NR, Lee JC, Lee HY et al (2014) An increase in the Akkermansia spp. population induced by metformin treatment improves glucose homeostasis in diet-induced obese mice. Gut 63: $727-735$

36. Gauffin Cano P, Santacruz A, Moya A, Sanz Y (2012) Bacteroides uniformis CECT 7771 ameliorates metabolic and immunological dysfunction in mice with high-fat-diet induced obesity. PLoS One 7:e41079

37. Morishita T, Tsutsui M, Shimokawa H et al (2005) Nephrogenic diabetes insipidus in mice lacking all nitric oxide synthase isoforms. Proc Natl Acad Sci U S A 102:10616-10621

38. Nakata S, Tsutsui M, Shimokawa H et al (2008) Spontaneous myocardial infarction in mice lacking all nitric oxide synthase isoforms. Circulation 117:2211-2223

39. Hattori Y, Suzuki M, Hattori S, Kasai K (2003) Globular adiponectin upregulates nitric oxide production in vascular endothelial cells. Diabetologia 46:1543-1549

40. Koh EH, Kim M, Ranjan KC et al (2010) eNOS plays a major role in adiponectin synthesis in adipocytes. Am J Physiol Endocrinol Metab 298:E846-E853
41. Bouskila M, Pajvani UB, Scherer PE (2005) Adiponectin: a relevant player in PPARgamma-agonist-mediated improvements in hepatic insulin sensitivity? Int J Obes 29(Suppl 1):S17-S23

42. Daval M, Foufelle F, Ferre P (2006) Functions of AMP-activated protein kinase in adipose tissue. J Physiol 574:55-62

43. Sniderman AD, Furberg CD, Keech A et al (2003) Apolipoproteins versus lipids as indices of coronary risk and as targets for statin treatment. Lancet 361:777-780

44. Andrikopoulos S, Blair AR, Deluca N, Fam BC, Proietto J (2008) Evaluating the glucose tolerance test in mice. Am J Physiol Endocrinol Metab 295:E1323-E1332

45. Windelov JA, Pedersen J, Holst JJ (2016) Use of anesthesia dramatically alters the oral glucose tolerance and insulin secretion in C57Bl/6 mice. Physiol Rep 4:e12824

46. Duplain H, Burcelin R, Sartori C et al (2001) Insulin resistance, hyperlipidemia, and hypertension in mice lacking endothelial nitric oxide synthase. Circulation 104:342-345

47. Matsuzawa Y, Funahashi T, Kihara S, Shimomura I (2004) Adiponectin and metabolic syndrome. Arterioscler Thromb Vasc Biol 24:29-33

48. Romeo GR, Lee J, Shoelson SE (2012) Metabolic syndrome, insulin resistance, and roles of inflammation - mechanisms and therapeutic targets. Arterioscler Thromb Vasc Biol 32:1771-1776

49. Ridaura VK, Faith JJ, Rey FE et al (2013) Gut microbiota from twins discordant for obesity modulate metabolism in mice. Science $341: 1241214$ 